\title{
Construct an Intelligent Evacuation Guidance System with Open System Architecture
}

\author{
Kun-Ming $\mathrm{Yu}^{1,}{ }^{1}$, Huan-Po Hsu ${ }^{2}$, Nien-En Chung ${ }^{1}$, Cheng-Chang Lien ${ }^{1}$, Shao-Tsai Cheng ${ }^{3}$, Ming-Yuan Lei ${ }^{4}$ and \\ Nancy Tsai ${ }^{4}$ \\ ${ }^{1}$ Department of Computer Science and Information Engineering, Chung Hua University, Hsinchu, Taiwan \\ ${ }^{2} \mathrm{Ph}$.D. Program in Engineering Science, Chung Hua University, Hsinchu, Taiwan \\ ${ }^{3}$ Department of Construction Management, Chung Hua University, Hsinchu, Taiwan \\ ${ }^{4}$ Architecture and Building Research Institute, Ministry of the Interior, New Taipei City, Taiwan \\ *Corresponding author
}

\begin{abstract}
Intelligent building and environment sensing technology are becoming more and more popular. Many office buildings use IoT to set up environment collection sensors to collect environmental information, however the way of evacuation inside still using traditional evacuation guidance equipment. Therefore, this study proposes an intelligent evacuation guidance system with open system architecture (IEGSOS), integrating with environment monitoring system and intelligent guidance equipment. The IEGSOS is developed based on the service-oriented architecture (SOA) and the data exchange technology is utilized to provide an integrated programming interface. By adopting the multimedia technology and integrating with the existing communication and guidance devices in environments, the optimal evacuation path is provided to the asylum in case of environmental crisis as a reference for safety evacuation at critical times.
\end{abstract}

Keywords-evacuation guidance system; service-oriented architecture; application programing interface; IoT

\section{INTRODUCTION}

With the rise of the smart building, environment collection sensors have been wildly used and distributed in every space of buildings. For example, it can carry out indoor perception utilizing the IoT technology, collect environmental information, and adjust the central control air conditioning equipment to maintain the comfort of environment $[2,7,8,11]$. However, when there is a fire in an intelligent environment, evacuees just only can accordance with traditional evacuation guidance equipment to escape although they are in the intelligent environment.

When the fire occurs, if the evacuation route is blocked, the evacuees cannot obtain immediate and safety reference for escape guidance. Therefore, this study proposes an intelligent evacuation guidance system with open system architecture (IEGSOS) which possesses an application interface and integrates with multi-perception. The integration of the existed environment monitoring system with the intelligent refuge guidance system is provided through the application interface and the dynamic link library. Then, through open interface, multimedia devices with communication capabilities or guiding devices can receive the result of evacuation path, and display the evacuation information for guidance.
This rest of the paper is organized as follows. Section 2 describes the theoretical concept of this paper. Section 3 introduces the architecture of the intelligent open-interface evacuation guidance system. And Section 4 put forward an implement case. The final section summaries the research and make a conclusion.

\section{RELATED WORKS}

\section{A. Indoor Evacuation}

Indoor guidance equipment is a necessary equipment for many people or an open space to install. When the emergency occurs, the indoor guidance equipment is the only indicator referring the export for evacuees. Thus, the evacuation device must be regularly maintained and updated to ensure it can play a guiding role in the critical moment and let the evacuees can escape from the environmental crisis quickly.

The guidance equipment often used in indoor is the escape indication device usually furnished in the skirting board or on the corner. The escape indication device makes use of the green arrow pointing in the direction of exports. With a standby power supply, when the environment is blackout, it can provide lighting and indicators continually as a reference for evacuees to escape. However, the evacuation routes in an environment change as places' characteristics change, the fixed guide equipment is unable to provide other evacuation routes.

Dynamic evacuation guidance equipment is a set of evacuation guidance equipment that can be cooperated with an intelligent evacuation guidance system for indoor guidance according to the evacuation route it calculated. If the environment's evacuation route needs to be changed, it can make a dynamic change of guidance device's direction through wired or wireless mode.

\section{B. Intelligent Evacuation Guidance System}

Intelligent evacuation guidance system is a set of environment evacuation guidance system applied in the indoor complex space. With intelligent environmental perception and evacuation route calculus and guidance functions, through the intelligent evacuation guidance system, a relative safe 
evacuation route in environmental emergency is calculated to guide evacuees to escape [1].

The intelligent evacuation guide system must have three layers of structure, which are intelligent sensing, computing and guiding [4].

1) Sensing layer is an architectural layer that focuses on data collection. The format of sensing type has no strict requirements. It can be designed according to the environmental requirements like temperature, humidity, luminance, carbon monoxide or carbon dioxide, crowd recognition count or other risk factor data. It can also be selected according to the needs of evacuation route computation. In addition, the communication interface that receives environmental data from environmental monitoring system can also be referred to as one of the types of intelligent perception $[9,10]$.

2) Computing layer is the information collection and calculation engine. It does environment calculus or path judgment on the basis of environmental data collected by intelligent sensing module. Through analysis on the results of intelligent computing, safety evacuation routes of the environment will be obtained and can be transmitted according to the form demanded.

3) Guiding layer transmits the evacuation route to the guidance equipment or guidance system, using wire or wireless communication protocol, to display, without a specific form, depending on the needs or forms of information transmission, so that evacuees can achieve safety guidance during the escape process.

\section{INTELLIGENT OPEN-INTERFACE EVACUATION GUIDANCE SYSTEM}

The environmental perception system is a sensing system installed by many intelligent buildings or open spaces to regulate environmental comfort. The environmental information can be collected and discriminate through sensors. However, when the environment is abnormal, the environmental sensing system will not carry out the initiative evacuation, so we must wait for the existing fire equipment to send the danger notice. The IEGSOS uses service-oriented architecture (SOA) [3] and the interface integration technology providing interface connections for integration with the existing environment perception system. By this way, the computing environment security path can be shared by the environmental information and evacuation guidance system, so that office buildings with environmental awareness system can also have the function of evacuation guidance.

The IEGSOS needs to handle the integration interface and the main data types of the calculus. It is necessary to design an application interface that integrates with the environment monitoring system.

Finally, complete content and organizational editing before formatting. Please take note of the following items when proofreading spelling and grammar:

\section{A. Application Interface Design}

Environment perception system mainly collects different information according to different needs, but most environmental perceptions usually have the following information as environmental data, including: temperature, humidity, luminance, carbon monoxide, carbon dioxide or PM2.5. The fire alarm station uses temperature and smoke to check environment safety. Therefore, IEGSOS use API interface to integrate environment monitoring system. The structure shown in FIGURE I.

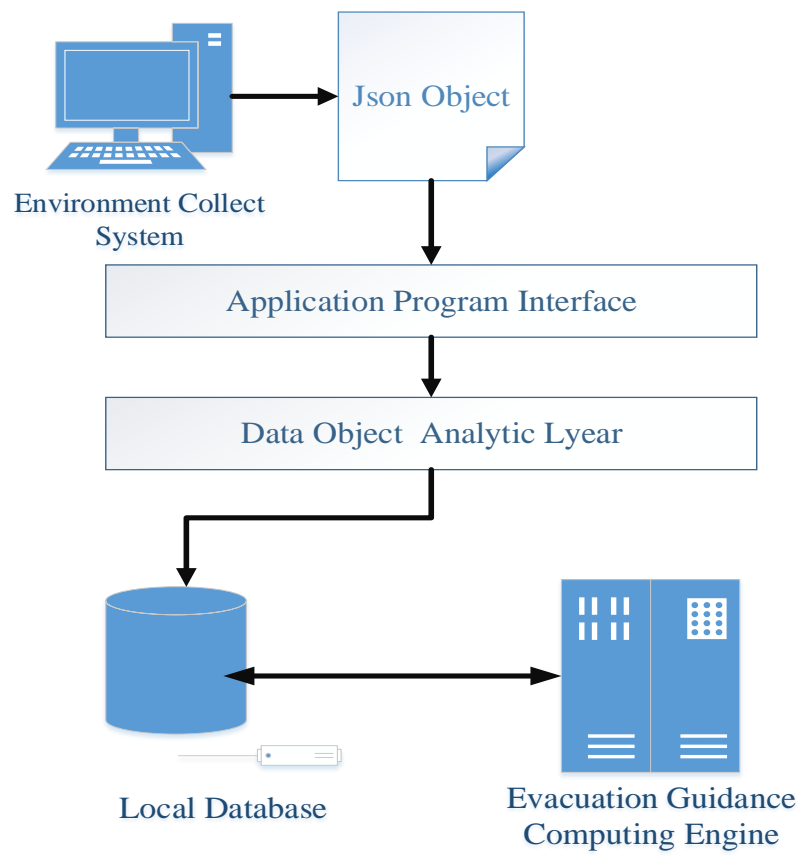

FIGURE I. IEGSOS INTEGRATE WITH ENVIRONMENT MONITORING SYSTEM THE INTERFACE STRUCTURE DIAGRAM.

According to FIGURE I, IEGSOS use JSON to interchange data, by which the transmission can be resolved in a fast and light way, and the data format can also be more pluralistic. The data needed in the evacuation guidance engine include environmental perception data and map information, while map information is presented in the form of environmental nodes, as shown in FIGURE II.

\section{B. Evacuation Planning Computing Engine}

Environmental data is transmitted by JSON objects. Data is decompressed and stored in the local database through data object analysis layer, to resolve the problem of wasting too much time when calculating database. To integrate the algorithm and the evacuation guidance system, crowd scatter guidance algorithm (DBCS) is used to do modularized modification so that the algorithm can easily invoke resources when required to call. 


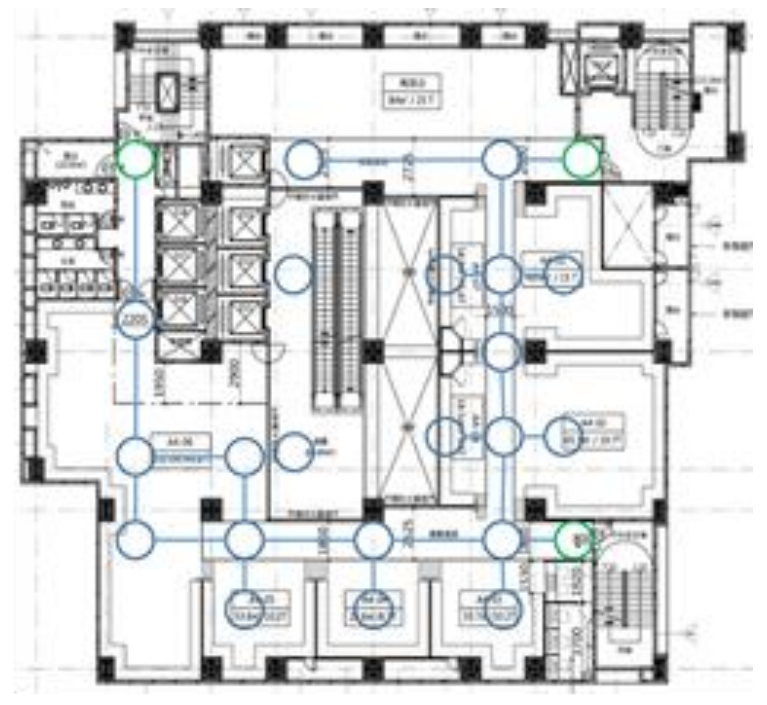

FIGURE II. THE MAP AND SET AREA NODE

\section{Evacuation Guidance Path Feedback}

When the data through the evacuation guidance engine, suggestions for indoor evacuation path will be put forward. These guidance path will be send out in the form of JSON, and the guidance system can receive and analyze the data through the interface, then do indoor evacuation guidance. The output in the form of JSON is shown in FIGURE III.

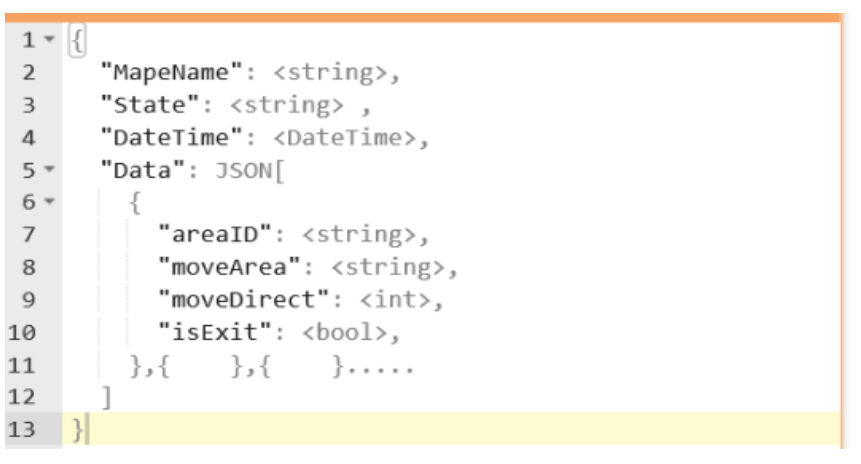

FIGURE III. EVACUATION PATH IN JSON FORMAT

\section{THE SYSTEM IMPLEMENTATION CASE}

In this study, integration test for the IEGSOS integrating the environmental perception system and the audiovisual evacuation system is described as follows [5, 6].

The environment perception system is a set of wide environmental information collection system adopting ZigBee transmission technology. The system collects temperature, humidity and illumination information of the environment. In order to know the sensor location in the environment, the system has an indoor environment map and the map information is constructed by the data interchange format JSON. The FIGURE IV is an integrated structure diagram of the environmental perception system with the open intelligent evacuation guidance system, and the FIGURE $\mathrm{V}$ is a diagram for JSON information.

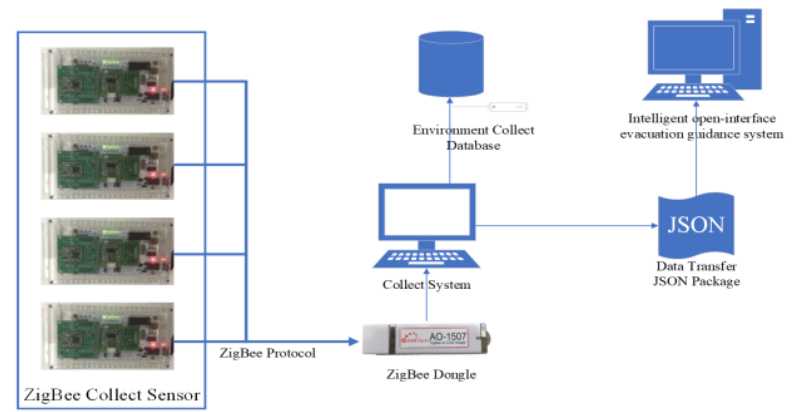

\section{FIGURE IV. ENVIRONMENT MONITORING SYSTEM AND IEGSOS STRUCTURE}

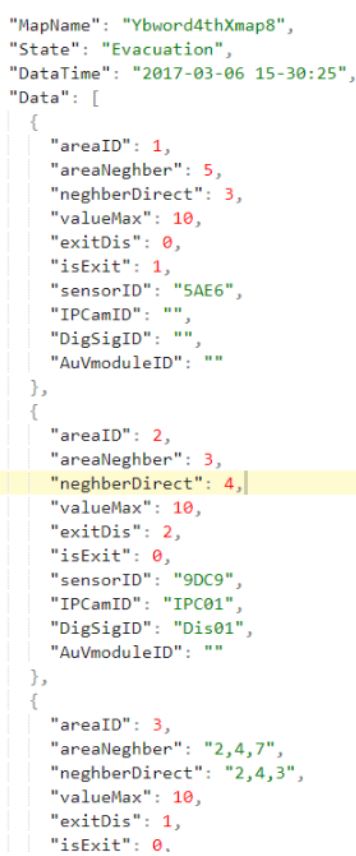

FIGURE V. ENVIRONMENT DATA JSON PACKAGE

IEGSOS receives callback data from the environment perception system through the JSON object, stores it to the database through the analytic layer, and extracts the database data by the evacuation guidance engine for algorithm calculation. Take advantage of the modular construction, the original two-dimensional time complexity algorithm is dismantled independently into multiple one-dimensional time complexity algorithms for simultaneous computing. Accordingly, even a multi-space building can be calculated in a short time, and the results are imported to the database for the storage of the evacuation path.

This study uses the actual site simulation. 22 space nodes are set according to the demand as shown in FIGURE VI. During the implementation, the temperature of node 14 in the environment perception system is increased by 50 degrees, making the sensing temperature abnormal, to initialize the IEGSOS. The operation result is shown in FIGURE VII. The operation results are transmitted in the form of JSON through the result feedback module, waiting for other guide systems to collect. The output results are shown in FIGURE VIII 


\section{CONCLUTION}

The IEGSOS proposed by this study is an integrated interface system based on the concept of diversification integration which can complete interface integration with the existing environment perception system, which possesses a high extensibility and provides guide command. By this way, it can integrate any existing intelligent devices meeting the needs by developing different integrated interfaces. It can integrate with existing environment system to reduce the cost of new system installation and provide the evacuation plan to the intelligent guidance equipment to guide evacuees to escape. Through simple translation, the guidance commands offered by the IEGSOS, through a simple translation, can be accepted by intelligent guidance devices, enabling the existing devices in the environment to become a part of the intelligent evacuation guidance system to make the intelligent environment safer.

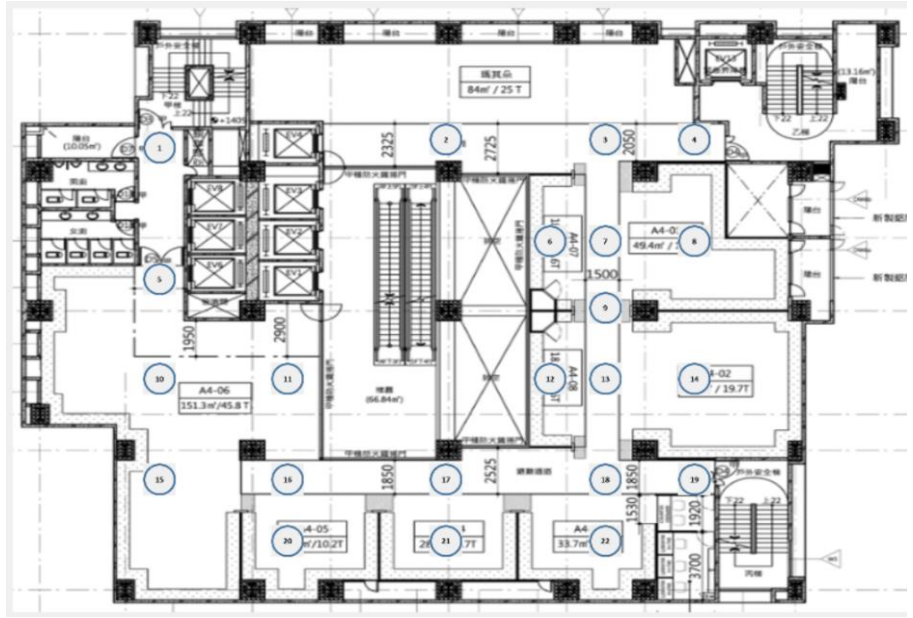

FIGURE VI. SET THE OUTPUT DIAGRAM OF THE ENVIRONMENT SPACE NUMBER

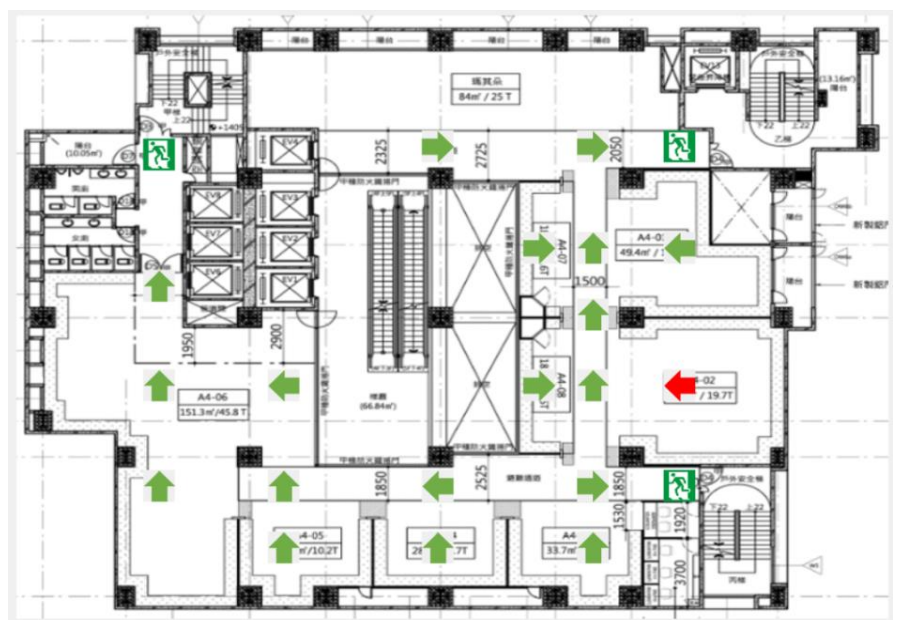

FIGURE VII. OUTPUT DIAGRAM OF ABNORMAL OF NODE 14(RED ARROW)

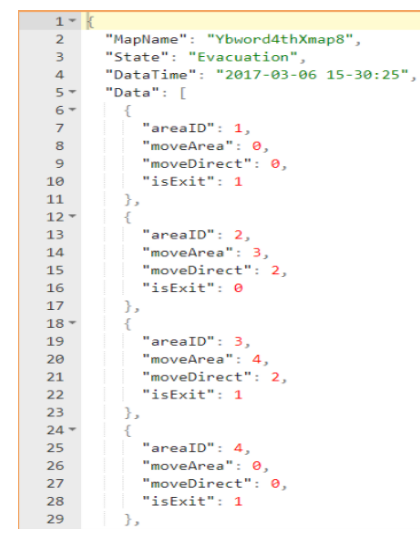

FIGURE VIII. Evacuation path feedback in JSON encapsulation

\section{ACKNOWLEDGMENT}

This paper was supported by Architecture and Building Research Institute, Ministry of the Interior (research No. 106301070000G0026).

\section{REFERENCES}

[1] Chung, N.-E., Yu, K.-M., Hsu, H.-P., Cheng, S.-T., Lien, C.-C., Lei, M.Y., \& Tsai, N. (2017). An Effectiveness Study of an Intelligent Emergency Evacuation System Using Field Verification Techniques. Sixth International Conference on Future Generation Communication Technologies (FGCT 2017) (pp. 96-101). Dublin, Ireland: IEEE.

[2] Cohen, B. (2012, 9 19). What Exactly Is A Smart City? Retrieved 3 12, 2017, from https://www.fastcoexist.com/1680538/what-exactly-is-asmart-city

[3] Fredlund, L. Å., Earle, C. B., Herranz, Á., \& Mariño, J. (2014). Property-Based Testing of JSON Based Web Services. 2014 IEEE International Conference on Web Services (pp. 704 - 707). Anchorage, AK, USA: IEEE.

[4] Hsu, H.-P. (2015). Design and Implement an Intelligent Evacuation Guiding System with Modular methodology. Hsinchu, Taiwan: Chung Hua University.

[5] Hsu, H.-P., Yu, K.-M., Chine, S.-T., Cheng, S.-T., Lei, M.-Y., \& Tsai, N. (2014). Emergency Evacuation Base on Intelligent Digital Signage Systems. 2014 7th International Conference on Ubi-Media Computing and Workshops (pp. 243-247). Ulaanbaatar, Mongolia: IEEE.

[6] Hsu, H.-P., Yu, K.-M., Lien, C.-C., Cheng, S.-T., Lee, C.-L., Lei, M.-Y., \& Tsai, N. (2016). An Intelligent Emergency Evacuation System Based on Multiple Guidance Technology. The 9th IEEE International Conference on Ubi-Media Computing (U-Media 2016) (pp. 397-402). Moscow, Russia: Science Index.

[7] IEEE. (2015, 12 17). IEEE 802.15 WPAN" ${ }^{\mathrm{TM}}$ Task Group 4 (TG4). Retrieved from IEEE 802.15: http://www.ieee802.org/15/pub/TG4.html

[8] ITU. (2005). ITU Internet Reports 2005: The Internet of Things. Retrieved from
https://www.itu.int/net/wsis/tunis/newsroom/stats/The-Internet-ofThings-2005.pdf

[9] Wu, C.-C. (2013). Development of a Personalized Crowd Guidance Algorithm for Emergency Fire Evacuation with Crowd Streaming Capability. Hsinchu, Taiwan: Chung Hua University.

[10] Yu, K.-M., Yu, C.-S., Lien, C.-C., Cheng, S.-T., Lei, M.-Y., Hsu, H.-P., \& Tsai, N. (2015). Intelligent evacuation system integrated with image recognition technology. 2015 8th International Conference on UbiMedia Computing (UMEDIA) (pp. 23-28). Colombo, Sri Lanka: IEEE.

[11] ZigBeeAlliance. (n.d.). Retrieved from ZigBee Alliance: http://www.zigbee.org/ 